\title{
Discussion section
}

Editor's Note: Written discussion about material published in the Bulletin is invited, and will be published, together with the Author's response, in this Section. Contributions other than Author's replies should reach the Editor not later than ten weeks before publication of the issue in which they are to appear.

\section{SHEPHARD AND GLOGAU- "SEISMIC DESIGN OF PUBLIC BUILDINGS"}

\author{
W. P. Edwards* and the Authors
}

\section{W. P. EDWARDS :}

The paper 'Seismic Design of Public Buildings', Shephard and Glogau - Bulletin Vol.2 No.4, gives valuable background information for users of the M.O.W. Code. These clear and helpful explanations for what might otherwise be regarded as arbitrary criteria will do much to improve designers' understanding and thus improve design.

Two matters seem worth further discussion.

1. Nothwithstanding the explanations in Paragraph 3, the exemption of single storey and the partial exemption of two to four storey buildings from ductility requirements seems less than rational. The claim that ductility demand in taller buildings will exceed that of the exempted buildings can only be made by ignoring things that ought not be disregarded. Among them:-

(a) For zone A buildings designed to the pseudo spectrum of NZSS 1900 Chapter 8, the low frequency responses are likely to be smaller multiples of the design figures than the high frequency responses are of design figures. This is readily seen from a comparison of the spectra in DSIR Bulletin 166 with the pseudo spectrum in NZSS 1900. Ductility demand is small when ratio design load to elastic response is large.

(b) Many New Zealand buildings regarded as 'tall', and the majority of intermediate height ( 5 to 15 storey buildings) will respond much more vigorously in their prime mode than in any other. If they are suitably detailed, there should be a reasonable spread of energy dissipation throughout the frame.

A case could thus be made that 'tall' buildings will have significantly higher levels of effective resistance than will 'small' buildings, when both are designed to the code. An exception must be made for Seismic Risk Zone $C$, where the question is complicated because the design pseudo spectrum is, erroneously, a constant sub multiple throughout

* Senior Partner, Edwards, Clendon and Partners, Wellington. its range of the Zone $A$ pseudo spectrum. However it is widely believed by those who reside in zone $\mathrm{C}$ that there is no risk there at all, so perhaps we can ignore such trivial anomolies?

Finally, on this question, it is interesting to compare SEAOC with NZSS 1900 Chapter 8 Loading requirements. They match approximately for small buildings, but much smaller capacities are required in california than in N.Z. for tall buildings. Remembering the relation between ductility and design load to elastic response ratio, we can conclude that the Californians would not agree with the ideas in paragraph 3 of the paper.

2. In paragraph 7 it is suggested that the under capacity factor for a shear core should change suddenly from 0.9 to 0.7 as the boundary of the compression block moves across the inner face of the transverse wall (refer to Fig. B10, page 408). This is a very arbitrary sort of abrupt change. Would it not be better to use provisions of ACI 318-63, Clause 1901 (c) which gives a better transition of under capacity factors?

\section{O. A. GLOGAU :}

Mr. Edwards' comments are welcome, particularly so in view of the scarcity of comment on the Ministry of Works code of practice from designers outside the department.

Mr. Edwards' first remark raises an interesting point regarding varying capacities resulting from the use of the New Zealand code. subject to the writer's comments below concerning the uncertainties in the shape of the pseudo spectrum and clarification of the term "low building" the writer agrees that Mr. Edwards" point should be considered together with those made by himself as favouring the low building. The graphs produced by Mr. Shephard on page 404 illustrates the same point and the reasons were discussed at the symposium at which this paper was first presented.

Perhaps it could be mentioned here that designers may avail themselves of clause 8.34 .4 
92

NZS1900. This tends in practice to reduce the lack of fit between the pseudo spectrum and code multiple since the required dynamic analysis is rarely carried out for very low buildings.

The matter goes, however, further than the mere comparison of code values and pseudo spectra. The designer as always must evaluate effects resulting from uncertainties in his assumption. Earthquakes may choose not to fit pseudo spectra on a given site, particularly a spectrum that has been derived from a site thousands of miles away and for lack of data compounded with scaled-up smaller ones. As yet we have not been "fortunate" enough to record a major disturbance in this country. Nevertheless there are already indications that we do not have to go to Almeda Park, Mexico, or zone $C$ to find sites with spectra having "humps" at longer periods than El Centro or our pseudo spectrum. Any such departure from our design spectrum would immediately reverse any presumed long period building advantages or render them fictional. On the other hand the short period building is insensitive to this uncertainty since it has already been designed for "hump" loadings.

Mr. Edwards has compared NZS 1900 chapter 8 loadings with SEAOC values. A number of modifications must be made, such as those for SEAOC " $K$ " factors, use of zero liveload by SEAOC, varying load factors and allowable code reductions used in practice in New Zealand, if we are to arrive at roughly comparable values. For the type and range of buildings the writer is discussing, he cannot confirm Mr. Edwards' observations. The " $r$ " values are some measure of the design overcapacities of zone A public buildings over SEAOC buildings. New Zealand 2 to 4 storey buildings $(r=2.2$ to 2.8$)$ have a greater or at least a comparable capacity as the N.Z. long period building of 1.2 to 2.0 seconds.

(The $r$ values in brackets are those where a designer of a long period building does not take advantage of a dynamic analysis and the allowable reduction to $80 \%$ code loading.) The writer does not take this as an indication that SEAOC are overly concerned about the very low building - and we do detail for ductility below 160 feet.

Finally in the course of this discussion the principal point seems to have been lost, namely how small a concession we are really making to the low buildings and how low they are. Let me reiterate here: buildings with Light roofs higher than 1 storey having beam hinge mechanisms and lightly loaded columns must be designed for full ductility excepting that for 2 storey public buildings (up to 3 storey and for a few occupancies up to 4 storey in zone c) we take a risk that an unforeseen column hinge could form. An unforeseen column hinge mechanism in a high building has a far more serious effect than one in a very low building.
In conclusion the ductility demand on a building depends on a combination of the various factors and others raised in this discussion. subject to clarification of the term "low" the writer believes that there is really no significant difference of opinion between himself and Mr. Edwards. There seems insufficient justification to extend full ductility requirements down to even lower buildings than those exempted at present - in general. 
R. B SHEPHARD:

In reference to note 2 :-

(i) There was no suggestion of a sudden change of under-capacityfactors,as the boundary of compression block moves across the inner face of the transverse wall. This could however be an inference from Fig. B10, which was intended to show the two extremes where the effect of a local compression failure, and hence the suggested under capacity, factors, were obvious. The text clearly stated the principle involved.

In the design the actual transition was made by using an under capacity factor of 0.9 until the compression block covered half of the transverse wall, (as in fig. $\mathrm{B} 10,(\mathrm{~b}))$, and to Iinearly reduce the value to 0.7 as the compression block depth increased to the inner face of the wall.

While this process of allocating under capacity factors may appear to be arbitrary in application, any more academic application would have limited value when all the unknown qualities of shear walls are taken into consideration. (ii) While the axial loads applied to the shear wall sections may be considered small with respect to the total section, it is felt that the shape of the section and the overall effect of a local compression failure must be considered when assessing the moment capacity. Further it is most important to take the axial loads into consideration, either the tensile load effect in reducing the moment capacity or to keep reinforcing to reasonable quantities where the moment capacity increases with applied axial load. It is interesting to note the provisions of the proposed revision of ACI 318-63, clause 9.2 where the modification of under capacity factors for members with small axial loads is more specifically stated. However, no allowance for the shape of a section is suggested. 